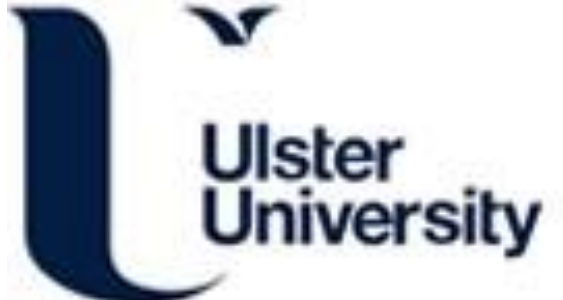

\section{Magnifying spontaneous facial micro expressions for improved recognition}

Sharma, P., Coleman, S., Yogarajah, P., Taggart, L., \& Samarasinghe, P. (2021). Magnifying spontaneous facial micro expressions for improved recognition. In Proceedings of ICPR 2020 - 25th International Conference on Pattern Recognition (pp. 7930-7936). [9412585] (Proceedings - International Conference on Pattern Recognition). Institute of Electrical and Electronics Engineers Inc..

https://doi.org/10.1109/ICPR48806.2021.9412585

Link to publication record in Ulster University Research Portal

Published in:

Proceedings of ICPR 2020 - 25th International Conference on Pattern Recognition

Publication Status:

Published (in print/issue): 10/01/2021

DOI:

10.1109/ICPR48806.2021.9412585

\section{Document Version}

Author Accepted version

\section{General rights}

Copyright for the publications made accessible via Ulster University's Research Portal is retained by the author(s) and / or other copyright owners and it is a condition of accessing these publications that users recognise and abide by the legal requirements associated with these rights.

\section{Take down policy}

The Research Portal is Ulster University's institutional repository that provides access to Ulster's research outputs. Every effort has been made to ensure that content in the Research Portal does not infringe any person's rights, or applicable UK laws. If you discover content in the Research Portal that you believe breaches copyright or violates any law, please contact pure-support@ulster.ac.uk. 


\section{Magnifying Spontaneous Facial Micro Expressions for Improved Recognition}

\author{
Pratikshya Sharma ${ }^{1}$, Sonya Coleman ${ }^{l}$, Pratheepan \\ Yogarajah $^{1}$, Laurence Taggart ${ }^{2}$ \\ School of Computing, Engineering \& Intelligent Systems ${ }^{1}$, \\ School of Nursing ${ }^{2}$ \\ University of Ulster \\ Northern Ireland \\ sharma-p3@ulster.ac.uk,sa.coleman@ulster.ac.uk, \\ p.yogarajah@ulster.ac.uk, 1.taggart@ulster.ac.uk
}

\author{
Pradeepa Samarasinghe \\ Department of Information Technology \\ Sri Lanka Institute of Information Technology \\ Sri Lanka \\ pradeepa.s@sliit.lk
}

\begin{abstract}
Building an effective automatic micro expression recognition (MER) system is becoming increasingly desirable in computer vision applications. However, it is also very challenging given the fine-grained nature of the expressions to be recognized. Hence, we investigate if amplifying micro facial muscle movements as a pre-processing phase, by employing Eulerian Video Magnification (EVM), can boost performance of Local Phase Quantization with Three Orthogonal Planes (LPQ-TOP) to achieve improved facial MER across various datasets. In addition, we examine the rate of increase for recognition to determine if it is uniform across datasets using EVM. Ultimately, we classify the extracted features using Support Vector Machines (SVM). We evaluate and compare the performance with various methods on seven different datasets namely CASME, CAS(ME) ${ }^{2}$, CASME2, SMIC-HS, SMIC-VIS, SMIC-NIR and SAMM. The results obtained demonstrate that EVM can enhance LPQ-TOP to achieve improved recognition accuracy on the majority of the datasets.
\end{abstract}

Keywords-Micro expression, EVM, LPQ-TOP

\section{INTRODUCTION}

For several years varying disciplines have aimed to understand human emotions and feelings, particularly for measuring health and well-being; communication is considered as a distinguished and competent tool for doing so. Classically, communication is categorized into two categories, verbal and non-verbal. Facial expressions are one such non-verbal tool that assist in comprehending a rich source of information essential for interpreting facial emotion and are broadly categorized into macro and micro expressions. Macro expressions have been extensively researched for more than three decades with exceptional results. Facial macro expressions are typically very prominent and can be determined very easily. Through various studies it has been observed that sometimes certain expressions are leaked on the face when a person tries to hide their emotions and are uncontrollable [7]. Such leaked expressions are commonly tagged as micro expressions [7]. Some experiments endorse the fact that by using automatic micro expression recognition (MER) techniques, we can achieve superior performance compared with manual approaches [11] [21] [27].
Both macro and micro expressions are highly informative, nonverbal cues in facial emotion analysis and hence examining such expressions has gained immense popularity over the last few decades. Compared with macro expression, micro expressions are not as well researched and are still an evolving area of interest. The two facial expressions differ mainly in terms of their duration. Research has shown that discriminative characteristics of facial micro expressions are its exceptionally short duration lasting approximately between 0.04 to 0.2 seconds [19] and remarkably low intensity muscle movements [7] [19]. Since facial muscle contractions last for a brief amount of time these facial movements are perceived to be almost invisible to the naked eyes and are likely to be missed using traditional observation methods. The main goal of ongoing research is to develop improved automated approaches for achieving superior facial MER accuracy using computer vision, deep learning and shallow learning techniques. Due to its diversified application areas, automatic recognition of facial micro expressions has increasingly become a research field of interest. This wide range of applications in the real world includes some of the critical and sensitive realms like criminal investigation, autism, psychology, schizophrenia, lie detection, business negotiations and mental diseases [8] [23].

In this research we are examining micro expressions for classifying facial emotion using computer vision and shallow learning techniques with an intent to extend its application on subjects with autism. A meta-analysis affirmed that people with Autism Spectrum Disorder (ASD) do not express facial expressions as explicitly as a typically developed (TD) individual [6]. Hence, people with ASD tend to possess very minimal ability to express facial expressions. The work in [6] also notes that these ASD related facial expressions have a short duration whereas [15] concludes that faces of individuals with ASD tend to have minimal facial muscle movements. These facial characteristics of individuals with autism are analogous to those of micro expressions and hence this research considers examining MER closely. Micro expressions are believed to expose legitimate emotions since they are natural, authentic, genuine and honest expressions [7][8]. Owing to less expressive 


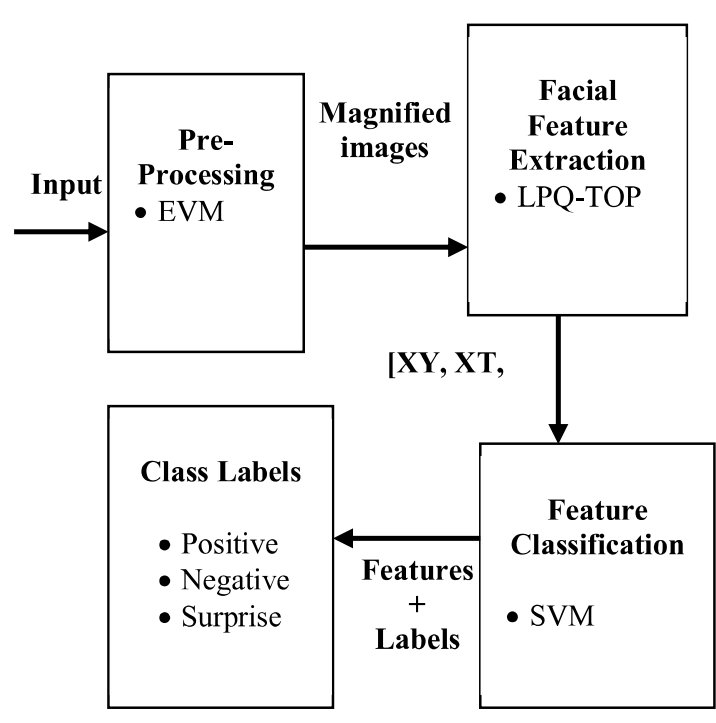

Fig. 1. Microexpression Recognition framework

capability of faces in individuals with autism, these faces occasionally fail to produce a wide range of expressions similar to TDs, hence as per our understanding classifying such expressions into three class labels, i.e. positive, negative and surprise, seems more realizable. Examining facial micro expressions in individuals with autism would significantly help in improving the ability to draw appropriate emotion implications and perform further analysis. Based on facial muscle contraction patterns, expressions are generally categorized as happy, sad, fear, surprise, disgust etc. Since micro expressions have inherently imperceptible characteristics, recognizing them is considerably challenging. There is a gradual increase in the volume of research work that has explored deep learning techniques for micro expressions but at present these works are less in number in comparison to hand crafted methods [5][12][14] [21]. One reason behind this is the well-known fact that deep learning techniques require significant data volume; thousands and millions of data samples. However, availability of data for facial micro expressions is far below the requirement. Nevertheless, researchers are developing diversified deep learning methods with an aim to cope with these deficiencies for providing promising future directions and positive outcomes.

In order to realize our concept for solving three class MER problem, initially facial micro features from magnified and nonmagnified frames are extracted using LPQ-TOP. Then these two sets of features are independently used as inputs into Support Vector Machines (SVM) for classification in the concluding step. In our work we have taken samples from seven different datasets. To keep our work on par with less expressive characteristics of faces with autism, samples from these datasets were relabeled as either positive, negative or surprise appropriately.

\section{RELATED WORK}

For building a competent MER framework it is crucial to use an effective feature extraction technique since it can enhance the performance of the overall recognition process. In view of this, we have surveyed some of the feature extraction techniques that have evolved in the past decade, particularly for extracting facial micro features. Research has revealed evolution of several variations of the hand crafted Local Binary Patterns (LBP). Initially Local Binary Patterns with Three Orthogonal Planes (LBP-TOP) was used as a baseline evaluation [29] and now it has been extended into new approaches like Local Binary Pattern with Six Intersection Points (LBP-SIP) and Local Binary Pattern with Mean Orthogonal Planes (LBP-MOP) [24]. Two variations using gradient approaches popularly known as Histogram of Gradient on Three Orthogonal Planes (HOG-TOP) and Histogram of Image Gradient on Three Orthogonal Planes (HIGO-TOP) have also been explored extensively for micro feature extraction [27]. Optical flow is another technique that is gaining popularity for MER related problems and has provided competent results but tends to be computationally expensive [13]. As previously noted, a progressive shift from these handcrafted, optical and gradient methods to deep learning techniques has been spotted in recent times in computer vision generally. In order to address the subtleties of micro expressions, recently video magnification has been identified as an effective technique and this is substantiated with experiments reporting increase in recognition accuracy with its usage [1] [23] [27]. Experiments also demonstrate that a Temporal Interpolation Model (TIM) combined with video/motion magnification help to promote capabilities of existing MER systems [1] [17] [20] [23] [27]. Taking inspiration from recent research, we employ Euler's Video Magnification (EVM) [10] technique for boosting MER. One of the well-known feature extraction methods, Local Phase Quantization (LPQ), was initially introduced for blurred texture analysis [16] [22] but today this concept has been extended for facial macro and micro [30] feature extraction.

In our previous work [18], we picked LPQ approach and applied it along three orthogonal planes, denoted as LPQ-TOP for extracting facial micro features. This method is revisited in our current research to demonstrate its usefulness with EVM, which had not been addressed in [18]. The contribution of our work is that it presents an extensive analysis to demonstrate the effectiveness of employing EVM with LPQ-TOP on a variety of datasets. As a second contribution it also provides an insight on the competency of LPQ-TOP as micro facial feature extraction technique in comparison to other existing popular techniques on a range of available spontaneous micro expression datasets.

\section{Methodology}

The general framework adopted for MER includes the following steps: pre-processing, feature extraction and feature classification [21]. In our experiments we have augmented a video magnification method and LPQ-TOP feature extraction technique on seven different datasets for achieving recognition results comparable with standard methods. Details of the framework used to tackle three class MER problem are illustrated in Fig 1. 

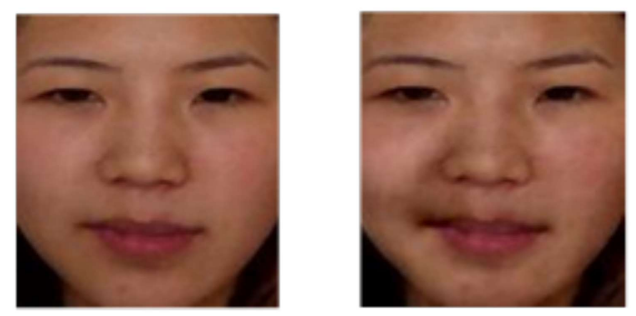

Fig. 2. Happy Expression[25], before(left) and after(right) magnification. (CXXiaolan Fu)

\section{A. Pre-processing}

Before initiating the actual micro expression classification process, as an initial step we perform pre-processing. This involves using EVM for magnifying and boosting the muscle motion intensity (refer Fig 2). The EVM method uses a unique spatio-temporal approach that does not require feature tracking or motion estimation for achieving motion magnification. Given a video sample, this technique uses two key steps for achieving magnification. Firstly, it performs spatial decomposition where the video is decomposed into spatial frequency of varying bands and secondly it uses temporal filtering for the frames, this is followed by amplifying the resultant signal. This magnification process is based on a phenomenon that is commonly used in optical flow, known as Taylor series expansion [10]. The fundamental idea of this method is to amplify motion without exclusively tracking the motion. In this proposed work the video magnification technique has been taken from [10] represented by (1). In equation (1), notation $\lambda$ represents the spatial wavelength, $\alpha$ is the magnification factor, $\delta(t)$ represents the observed motion. The magnification level can be regulated by varying the $\alpha$ parameter in (1). In our experiment the datasets have been magnified by setting the magnification factor $\alpha=26$ which is closer to the value 20 used to achieve $75.3 \%$ accuracy in [23]. Figure 2 depicts an instance of happy micro expression from CASME dataset after employing magnification. The final magnified image frames obtained are then used in the feature extraction step implemented using LPQ-TOP.

$$
(1+\alpha) \delta(t)<\frac{\lambda}{8}
$$

\section{B. Facial Feature Extractionns}

The extracted facial micro features form the foundation for recognition in the later stage. Building on our previous work [18], we are continuing to explore the LPQ-TOP method. We use this technique for extracting facial micro features from image sequences that have been magnified as in Section IIIA. It is a well-established fact that the LPQ method revolves around the idea of performing quantization of Fourier transforms. For spatially invariant blurring, an original image $f(\mathbf{x})$ that results in an observed image $\mathrm{g}(\mathbf{x})$ can be represented using convolution [16] [22], expressed as:

$$
\mathrm{g}(\mathbf{x})=(f * h)(\mathbf{x})
$$

where $h(\mathbf{x})$ denotes point spread function (PSF), * notation represents 2-D convolution and $\mathbf{x}$ represents a vector of coordinates, denoted by $[x, y]^{T}$. This expression can be represented in the Fourier domain [16] [22] as:

$$
G(\mathbf{u})=F(\mathbf{u}) \cdot H(\mathbf{u})
$$

where $G(\mathbf{u})$ is the Discrete Fourier transform (DFT) of the blurred image, $F(\mathbf{u})$ represents the DFT of the original image and $H(\mathbf{u})$ represents DFT of the PSF. In addition, $\mathbf{u}$ is a vector of coordinates, denoted by $[u, v]^{T}$. Since this method considers only the phase, the equation can be expressed in terms of a sum [16] [22]:

$$
\angle G(\mathbf{u})=\angle F(\mathbf{u})+\angle H(\mathbf{u})
$$

where $\angle G(\mathbf{u}), \angle F(\mathbf{u})$ and $\angle H(\mathbf{u})$ represent the phase angle for $G(\mathbf{u}), F(\mathbf{u})$ and $H(\mathbf{u})$ respectively. Considering $h(\mathbf{x})$ is centrally symmetric, i.e. $h(\mathbf{x})=h(-\mathbf{x})$, the Fourier transform will always be a real value and as a result the phase is reduced into a twovalued function [16] [22] represented as:

$$
\angle H(\mathbf{u})= \begin{cases}0 & \text { if } H(\mathbf{u}) \geq 0 \\ \pi & \text { if } H(\mathbf{u})<0\end{cases}
$$

This eventually results in:

$$
\angle G(\mathbf{u})=\angle F(\mathbf{u}) \text { for all } \angle H(\mathbf{u}) \geq 0
$$

In order to examine the phase, this method considers a local $\mathrm{M}$ $\mathrm{x}$ M neighborhood represented by the notation $N_{x}$ at each pixel position, $\mathbf{x}$, for a given image $f(\mathbf{x})$. A short-term Fourier transform (STFT) is computed as:

$$
F(\mathbf{u}, \mathbf{x})=\sum_{\mathbf{y} \in \mathrm{N}_{\mathbf{x}}} f(\mathbf{x}-\mathbf{y}) e^{-\mathrm{j} 2 \pi \mathbf{u}^{T} \mathbf{y}}
$$

Using 1-D convolutions consecutively for rows and columns, the STFT is evaluated for all the positions in a given image. It computes local Fourier coefficients at each pixel location for four frequency points; $\mathbf{u}_{1}=[a, 0]^{T}, \mathbf{u}_{2}=[0, a]^{T}, \mathbf{u}_{3}=[a, a]^{T}$, $\mathrm{u}_{4}=[a,-a]^{T}$. Here, " $a$ " represents a scalar frequency that satisfies $H(\mathbf{u}) \geq 0$. This ultimately results in a vector for each pixel position that can be expressed as given in (8), where $R e$ represents the real and Im represents the imaginary parts respectively [16] [22].

$$
\begin{gathered}
F(\mathbf{x})=\left(\left[\operatorname{Re}\left\{F\left(\mathbf{u}_{1}, \mathbf{x}\right)\right\}, \operatorname{Im}\left\{F\left(\mathbf{u}_{1}, \mathbf{x}\right)\right\}\right], \ldots\right. \\
\left.\ldots,\left[\operatorname{Re}\left\{F\left(\mathbf{u}_{4}, \mathbf{x}\right)\right\}, \operatorname{Im}\left\{F\left(\mathbf{u}_{4}, \mathbf{x}\right)\right\}\right]\right)
\end{gathered}
$$

The next step is calculating the phase information. To do so binary scalar quantizer shown in (9), is used and quantizes the signs of real and imaginary parts of the coefficients obtained in the previous step [16] [22]. In equation (9), $f_{j}$ represents the $j^{\text {th }}$ component for a given vector $F(\mathbf{x})$. The resultant eight-bit binary coefficients are represented in the form of integers using the binary coding technique. It is then followed by mapping the 
eight-bit binary into one of the 256 bin histograms, i.e. the resulting codes are represented in the form a histogram.

$$
q_{j}= \begin{cases}1 & \text { if } f_{j} \geq 0 \\ 0 & \text { if } f_{j}<0\end{cases}
$$

The LPQ technique ideally works for static images however, we are examining sequence of images and hence are extending this concept to include the time domain, denoted as LPQ-TOP. Therefore, we extract all the features along the XY, XT and YT planes, commonly referred as three orthogonal planes. The descriptors extracted from each of these planes are represented in the form of a histogram and also each of these histograms are concatenated in the final steps to form a single feature descriptor. Although [30] explored the concept of LPQ-TOP for micro expressions, our work differs from it in three ways. Firstly, we do not focus on designing a cross-database rather our focus is on improving recognition accuracy for solving classification problem. Secondly, we are using a magnification process which has not been probed in [30] for solving three class labels. Thirdly, we have adopted more datasets than any other experiment and hence demonstrate the robustness of our approach. Following the work in [27][30] we have employed the LPQ-TOP feature extraction method for micro expression in conjunction with EVM.

\section{Feature Classification}

The final feature vector obtained from images after undergoing feature extraction is used to train a Support Vector Machine (SVM) [4]. It is a well-known fact that SVM is a supervised machine learning algorithm typically used as a feature classifier essentially due to its discriminative nature by exploiting optimal hyperplanes to categorize data. SVM is said to be effective for small datasets with higher dimensions hence, this classification method was chosen for our work as the datasets and method employed match these criteria. Experiments for classification were carried out using three different kernels namely polynomial, radial basis function (RBF) and linear. During the experiment it was observed that the linear kernel worked best for the SMIC [28] and CASME2 [29] datasets whereas RBF produced the highest accuracy on the CASME [25] dataset. The performance of SVM using a polynomial kernel provided comparatively better accuracy for the CAS(ME) ${ }^{2}$ [9] and SAMM [2][3] datasets. As we are focusing on categorizing facial micro expression into three classes, we have chosen positive, negative and surprise labels. Also, throughout our experiment all happy expressions have been given positive label and surprise expressions are left unchanged. Expressions like fear, anger, sadness, tense and disgust are categorized under negative label. Data samples with expressions labeled as pain, helpless, contempt, repressions and others have not been considered in this work in an attempt to maintain a close uniformity in data labels across all datasets used. The data samples considered for this work have been taken from seven different micro expression (ME) database as discussed in Section IV.

\section{EXPERIMENTS, RESULTS AND DISCUSSION}

The seven different datasets considered for our experiments were Chinese Academy of Sciences Micro-expression (CASME [25], CAS(ME) ${ }^{2}$ [9], CASME2 [29]); Spontaneous Microexpression database (SMIC-HS, SMIC-VIS, SMIC-NIR) [28] and Spontaneous Micro-Facial Movement Dataset (SAMM [2][3]). SMIC-HS (High Speed) datasets contain samples recorded at a rate of 100 frames per second (fps), whereas SMIC-VIS (Visual) and SMIC-NIR (Near Infrared) are recorded at $25 \mathrm{fps}$. Total count of samples used were 164, 71 and 71 from SMIC-HS, SMIC-VIS and SMIC-NIR respectively. A total of 133 samples from CASME datasets recorded with 60fps were also used. CAS(ME) ${ }^{2}$ dataset consists of samples with both macro and micro expressions and we have used 51 expressions that were labeled as micro expressions recorded at $30 \mathrm{fps}$. Correspondingly 122 data samples were used from the CASME2 dataset and 121 micro expression data samples were used from the SAMM dataset. Samples taken from the CASME2 and SAMM databases were recorded with $200 \mathrm{fps}$ and are two datasets that have good facial spatial resolution compared with the other datasets. The experiments conducted were divided into two phases. In the first phase features were extracted from raw (non-magnified) data samples using the LPQ-TOP method. Applying a suitable neighborhood size is crucial with the LPQTOP technique. In our experiment, for YT plane a neighborhood size of 5 has been used. Similarly, on XY and XT planes a neighborhood size of 5 has been considered. Keeping the abovementioned parameters intact the second phase of experiment was also conducted. Here samples were first magnified using EVM with a magnification factor set to 26 (refer Fig 2), then the features were extracted using LPQ-TOP. By extracting features in the above two phases we obtained two feature vectors. Two instances of SVM, one for each vector type has been taken into consideration for training and classifying data samples into one of the three labels i.e. negative, positive and surprise. Here, 10fold cross validation for training along with multi-class classification using one-vs-all strategy was considered. Table I summarizes the accuracy results obtained for micro expression classification before and after introducing the magnification with LPQ-TOP for all seven datasets. From Table I, it is evident that magnification has a significant impact on the performance accuracy for LPQ- TOP which is demonstrated by an increase in the recognition accuracy among all datasets. However, it must be noted that although an enhancement in recognition performance after magnification is evident, there is an absence of uniformity on the rate of enhancement on all seven datasets. For example, an increase of $13.34 \%$ in the accuracy has been observed for the CASME2 dataset after introducing magnification which is a significant rise in comparison to only $1.67 \%$ improvement using the SAMM dataset. Both of these datasets contain samples recorded at $200 \mathrm{fps}$ with approximately the same number of samples considered for our experiment, yet the effect of magnification varies significantly.

TABLE I. ACCURACY\% USING LPQ-TOP 


\begin{tabular}{|l|c|c|c|}
\hline \multirow{2}{*}{ Dataset } & \multicolumn{3}{|c|}{ Our Accuracy \% (LPQ-TOP) } \\
\cline { 2 - 4 } & No Magnification & $\begin{array}{c}\text { With } \\
\text { Magnification }\end{array}$ & $\begin{array}{c}\text { \% } \\
\text { Increase }\end{array}$ \\
\hline CASME & 83.45 & 88.2 & 4.75 \\
\hline CASME2 & $61.16[18]$ & 74.5 & 13.34 \\
\hline CAS(ME) & 63.6 & 68.5 & 4.9 \\
\hline SAMM & 70.4 & 72.07 & 1.67 \\
\hline SMIC-VIS & 65.6 & 73.8 & 8.2 \\
\hline SMIC-NIR & 63.3 & 70.42 & 7.12 \\
\hline SMIC-HS & 62.8 & 65.8 & 3 \\
\hline
\end{tabular}

In contrast, the rate of increase using SMIC-NIR and SMICVIS differs by approximately $1 \%$. This means the method has uniform increase in recognition accuracy on these two SMIC datasets. Interestingly, these two SMIC datasets also share equal numbers of samples along with same rate of fps, which is similar to CASME2 and SAMM yet, the results for these two scenarios vary significantly. It is unclear as to what led to this variation and this may require further investigation. It must be noted that the distribution of data samples across the three classes is almost uniform, i.e., balanced, in the SMIC dataset. This could have given an advantage to the classification technique to achieve uniform increase in recognition accuracy. From the results in Table I an average increase in recognition accuracy of $\sim 6.14 \%$ across all datasets is achieved using the novel framework to solve three class MER problem. The accuracy achieved after introducing magnification with the CASME, CASME2, CAS(ME) ${ }^{2}$, SAMM and SMIC datasets in comparison to other methods is presented in Table II. These results demonstrate our proposed approach outperforms the performance accuracy of many of the other approaches on the various datasets. The approach used in our work is also able to produce accuracy higher than Histogram of Gradient with magnification (HOG + mag) and LBP-TOP (with magnification) on SMIC-HS and CASME2 dataset but is slightly less efficient than Histogram of Image Gradient Orientation (HIGO $+\mathrm{mag}$ ). Therefore, the results of our proposed approach look promising and are closely comparable with these three methods. The LPQ-TOP method has achieved an accuracy of $73.8 \%$ using the SMIC-VIS data but the performance falls short in comparison to accuracy values reported using other methods. A significantly improved recognition accuracy of $88.2 \%$ is obtained for CASME and is the highest accuracy achieved using the LPQ-TOP with EVM compared to all other datasets. In our experiment we have excluded some of the samples, this may have given an added advantage to our chosen method thus resulting in high recognition accuracy. The method also produces good accuracies of $68.5 \%$ and $72.07 \%$ on the CAS(ME) $)^{2}$ and SAMM datasets respectively. From these results we can conclude that the performance of LPQ-TOP with magnification is comparable methods like NMP (Necessary Morphological Patches) [31] and Convolution Neural Network (CNN) [32] on CAS(ME) ${ }^{2}$ and SAMM respectively.

TABLE II. ACCURACY\% COMPARISON FOR CASME, CASME2, $\mathrm{CAS}(\mathrm{ME})^{2}, \mathrm{SAMM} \& \mathrm{SMIC}$
A close analysis of these results shows that while magnification

\begin{tabular}{|c|c|c|c|}
\hline \multirow{2}{*}{ Dataset } & \multirow{2}{*}{$\begin{array}{c}\begin{array}{c}\text { Our } \\
\text { Work }\end{array} \\
\text { LPQ- } \\
\text { TOP+ } \\
\text { mag }\end{array}$} & \multicolumn{2}{|c|}{ Other Authors } \\
\hline & & $\begin{array}{c}\text { Accuracy } \\
\%\end{array}$ & Method \\
\hline CASME & 88.2 & 80.2 & MMPTR [26] \\
\hline \multirow{3}{*}{ CASME2 } & \multirow{3}{*}{74.5} & 78.14 & HIGO + mag [27] \\
\hline & & 63.97 & $\mathrm{HOG}+\mathrm{mag}[27]$ \\
\hline & & 60.73 & LBP-TOP + mag [27] \\
\hline $\mathrm{CAS}(\mathrm{ME})^{2}$ & 68.5 & 64.07 & NMP [31] \\
\hline SAMM & 72.07 & 70.18 & CNN [32] \\
\hline \multirow{3}{*}{ SMIC-VIS } & \multirow{3}{*}{73.8} & 81.69 & HIGO + mag [27] \\
\hline & & 77.46 & $\mathrm{HOG}+\mathrm{mag}[27]$ \\
\hline & & 78.87 & LBP-TOP + mag [27] \\
\hline \multirow{3}{*}{ SMIC-NIR } & \multirow{3}{*}{70.42} & 67.61 & HIGO + mag [27] \\
\hline & & 64.79 & $\mathrm{HOG}+\operatorname{mag}[27]$ \\
\hline & & 67.61 & LBP-TOP + mag [27] \\
\hline \multirow{3}{*}{ SMIC-HS } & \multirow{3}{*}{65.8} & 68.29 & HIGO + mag [27] \\
\hline & & 61.59 & $\mathrm{HOG}+\operatorname{mag}[27]$ \\
\hline & & 60.37 & LBP-TOP + mag [27] \\
\hline
\end{tabular}

has a profound effect on the CASME2 dataset, LPQ-TOP works significantly well on the CASME dataset. Thus, we can say that the overall performance of this approach is competitive enough to be compared and contrasted with other methods.

\section{CONCLUSION}

Through this work we have presented a comprehensive performance analysis involving seven different spontaneous micro expression datasets to reach a useful conclusion that LPQ-TOP when fused with EVM shows an impressive performance boost on some datasets and is useful for dealing MER problems but lacks an orderly increase of recognition accuracy. The results obtained have achieved an impressive average increase of $\sim 6.14 \%$ considering all seven ME datasets. This strengthens the common belief that magnification helps extraction techniques to efficiently extract required facial micro features thereby boosting recognition performance to a large extent. A further investigation is required to understand the bias towards the CASME2 dataset after magnification in comparison to other datasets sharing similar data count and fps. In addition, this work has successfully realized performance comparison between LPQ-TOP (with and without EVM) and various other approaches on seven different spontaneous micro expression datasets. Evidently LPQ-TOP technique is as competent as other hand-crafted methods and can be readily exploited as a facial micro feature extraction technique. Results obtained provide a good guideline for future comparisons particularly for CASME2, CASME and SMIC-NIR datasets. The ultimate goal of performing this work is to provide a novel pipeline for solving three class MER problem. As mentioned previously MER is still evolving and has wide scope in multidisciplinary areas, one such potential area is autism. Since, the final goal of our work is to extend MER for autism 
screening, detection and diagnosis, our current work is at its initial stage and we have achieved high recognition results comparable with other approaches. In order to perform competent micro expression analysis, having an adequate number of training samples is crucial and this is often a challenge due to unavailability of adequate volume of spontaneous micro expression data samples and datasets. Imbalanced classification is one of the limitations of our current approach due to an unequal distribution of available data samples with an exception of SMIC datasets, this problem will be addressed in future to achieve more balanced and robust classification results.

\section{REFERENCES}

[1] A. C. Le Ngo, Y. Oh, R. C. -. Phan and J. See, "Eulerian emotion magnification for subtle expression recognition," 2016 IEEE International Conference on Acoustics, Speech and Signal Processing (ICASSP), Shanghai, pp.1243-1247, 2016

[2] A. K. Davison; C. Lansley; N. Costen; K. Tan; M. H. Yap, "SAMM: A Spontaneous MicroFacial Movement Dataset," in IEEE Transactions on Affective Computing, vol. 9, no. 1, pp. 116- 129,2018

[3] A.K. Davison, W. Merghani and M.H. Yap, "Objective classes for microfacial expression recognition”, Journal of Imaging, 4(10), p.119, 2018.

[4] Chih-Chung Chang and Chih-Jen Lin, LIBSVM: a library for support vector machines. ACM Transactions on Intelligent Systems and Technology, vol. 2 no. 3 , p. 27,2011

[5] Devangini Patel, X. Hong and G. Zhao, "Selective deep features for microexpression recognition", 23rd International Conference on Pattern Recognition (ICPR), Cancun, pp. 2258-2263, 2016.

[6] Dominic A. Trevisan, Maureen Hoskyn, Elina Birmingham, "Facial Expression Production in Autism", Autism Research 11:, International Society for Autism Research, Wiley Periodicals, Inc.,1586-1601, 2018.

[7] Ekman P, Friesen WV, "Nonverbal leakage and clues to deception", Psychiatry 32, pp. 88-106, 1969.

[8] Ekman P, "Telling Lies: Clues to Deceit in the Marketplace, Politics, and Marriage”, (Revised Edition), WW Norton \& Company, 2009.

[9] F. Qu, S. J. Wang, W.J. Yan, H. Li, S. Wu and X. Fu, “CAS (ME)^ 2: a database for spontaneous macro-expression and micro-expression spotting and recognition," IEEE Trans. Affect. Comput., 2017.

[10] Hao-Yu Wu, Michael Rubinstein, Eugene Shih, John Guttag, Frédo Durand, and William Freeman."Eulerian video magnification for revealing subtle changes in the world”. ACM Trans. Graph. 31, 4, Article 65, July 2012.

[11] J. Endres and A. Laidlaw, "Micro-expression recognition training in medical students: a pilot study", BMC medical education, vol. 9 , no. 1 , p. 47 , 2009.

[12] Liong, Sze-Teng et al. "Shallow Triple Stream Three-Dimensional CNN (STSTNet) for Micro-Expression Recognition." 14th IEEE International Conference on Automatic Face \& Gesture Recognition (FG 2019), 2019.

[13] Liu, Y.J., Zhang, J.K., Yan, W.J., Wang, S.J., Zhao, G., Fu, X.,” A main directional mean optical flow feature for spontaneous micro- expression recognition”. IEEE Trans. Affect Comput. 7(4),299-310, 2016.

[14] M. A. Takalkar and M. Xu, "Image Based Facial Micro-Expression Recognition Using Deep Learning on Small Datasets," International
Conference on Digital Image Computing: Techniques and Applications (DICTA), Sydney, NSW, pp. 1-7. 2017.

[15] P. Czapinski, S.E. Bryson, "Reduced facial muscle movements in autism: Evidence for dysfunction in the neuromuscular pathway?", Brain and Cognition, 51(2), 177-179, March 2003

[16] Päivärinta J., Rahtu E., Heikkilä J., "Volume Local Phase Quantization for Blur- Insensitive Dynamic Texture Classification”. In: Heyden A., Kahl F. (eds) Image Analysis. SCIA 2011. Lecture Notes in Computer Science, vol 6688. Springer, Berlin, Heidelberg, pp. 360-369, 2011.

[17] Peng, Wei et al. "A Boost in Revealing Subtle Facial Expressions: A Consolidated Eulerian Framework." 14th IEEE International Conference on Automatic Face \& Gesture Recognition (FG 2019), 2019.

[18] Sharma, P., Coleman, S., Yogarajah, P. \& Laurence, T., "Micro expression classification accuracy assessment". IMVIP 2019: Irish Machine Vision \& Image Processing, Technological University Dublin, Dublin, Ireland, August 28-30, 2019

[19] Sze-Teng Liong, John See, KokSheik Wong, Raphael C.-W. Phan,"Less is more: Micro-expression recognition from video using apex frame", Elsevier, pp 0923-5965, 14 December 2017.

[20] T. Pfister, Xiaobai Li, G. Zhao and M. Pietikäinen, "Recognising spontaneous facial micro-expressions," International Conference on Computer Vision, Barcelona, pp. 1449-1456, 2011

[21] Takalkar, M., Xu, M., Wu, Q. et al. "A survey: facial micro-expression recognition”. Multimed Tools Appl 77, 19301-19325, 2018

[22] V. Ojansivu and J. Heikkilä, "Blur insensitive texture classification using local phase quantization", International conference on image and signal processing. Springer, pp. 236-243, 2008.

[23] Wang, Y., See, J., Oh, Y. et al., "Effective recognition of facial microexpressions with video motion magnification". Multimed Tools Appl 76, pp 21665-21690, 2017.

[24] Wang Y., See J., Phan R.CW., Oh YH (2015), "LBP with Six Intersection Points: Reducing Redundant Information in LBP-TOP for Micro-Expression Recognition". In: Cremers D., Reid I., Saito H., Yang MH. (eds) Computer Vision -- ACCV 2014, Lecture Notes in Computer Science, vol 9003. Springer, Cham, 2014

[25] Wen-Jing Yan, Q. Wu, Yong-Jin Liu, Su-Jing Wang and X. Fu, "CASME database: A dataset of spontaneous micro-expressions collected from neutralized faces,"10th IEEE International Conference and Workshops on Automatic Face and Gesture Recognition (FG), Shanghai,pp. 1-7, 2013.

[26] X. Ben, P. Zhang, R. Yan, M. Yang, and G. Ge, "Gait recognition and micro-expression recognition based on maximum margin projection with tensor representation," Neural Computing and Applications, vol. 27, no. 8, pp. 2629-2646, 2016.

[27] Xiaobai Li, Xiaopeng Hong, Antti Moilanen, Xiaohua Huang, Tomas Pfister, Guoying Zhao, and Matti Pietikainen, "Towards Reading Hidden Emotions:A Comparative Study of Spontaneous Micro-Expression Spotting and Recognition Methods", IEEE Transactions On Affective Computing, Vol. 9, No. 4, October-December 2018 .

[28] Xiaobai Li; Pfister, T.; Xiaohua Huang; Guoying Zhao; Pietikainen, M., "A Spontaneous Micro-expression Database: Inducement, collection and baseline," in Automatic Face and Gesture Recognition (FG), 2013 10th IEEE International Conference and Workshops on, vol., no., pp.1-6, 22-26 April 2013.

[29] Yan W-J, Li X, Wang S-J, Zhao G, Liu Y-J, et al., "CASME II: An Improved Spontaneous Micro-Expression Database and the Baseline Evaluation”, PLoS ONE 9(1): e86041, 2014. 
[30] Yuan Zong, Wenming Zheng, Xiaopeng Hong, Chuangao Tang, Zhen Cui, Guoying Zhao, "Cross-Database Micro-Expression Recognition: A Benchmark". CoRR abs/1812.07742, 2018.

[31] Zhao, Y., \& Xu, J., “An Improved Micro-Expression Recognition Method Based on Necessary Morphological Patches". Symmetry, 11, 497, 2019.

[32] Zhao Y, Xu J. "A Convolutional Neural Network for Compound MicroExpression Recognition”. Sensors.19(24):5553, 2019. 